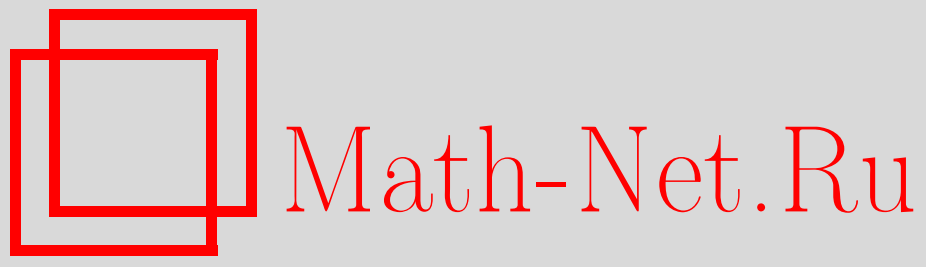

А. Н. Паршин, Соответствие Кричевера для алгебраических поверхностей, Функи. анализ и его прил., 2001, том 35, выпуск 1, 88-90

DOI: https://doi.org/10.4213/faa237

Использование Общероссийского математического портала MathNet.Ru подразумевает, что вы прочитали и согласны с пользовательским соглашением

http://www.mathnet.ru/rus/agreement

Параметры загрузки:

IP : 54.166 .219 .16

26 апреля 2023 г., 04:56:38

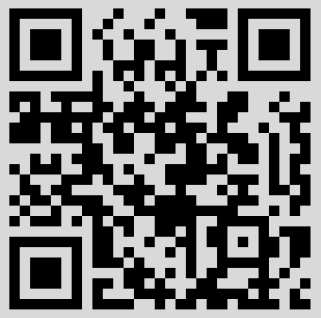


УДК 512.7

\title{
Соответствие Кричевера для алгебраических поверхностей
}

\author{
(c) 2001. А. Н. ПАРШИН
}

В теории интегрируемых систем известна конструкция И. М. Кричевера, сопоставляющая алгебраическим кривым и векторным расслоениям на них бесконечномерное подпространство в пространстве $k((z))$ степенных рядов Лорана [6]. Эта конструкция имеет многочисленные применения к уравнениям типа КП и к теории модулей алгебраических кривых $[6,14,2,7,1,13,3]$.

Недавно автор заметил $[11,10]$ некоторые связи между уравнениями КП и $n$-мерными локальными полями $[9,5,4]$. Основываясь на этой связи, мы предлагаем обобщение конструкции Кричевера для алгебраических поверхностей. Сначала опишем эту конструкцию для случая алгебраических кривых.

Пусть $\mathscr{M}_{1}=\left\{\left(C, P, z, \mathscr{F}, e_{P}\right)\right\}$, где $C-$ проективная неприводимая кривая над полем $k, P \in C$ - гладкая точка, $z-$ формальный локальный параметр в $P$, $\mathscr{F}-$ пучок без кручения ранга $r$ на $C$ и $e_{P}-$ тривиализация пучка $\mathscr{F}$ в $P$. Независимо мы имеем поле $k((z))$ и пространство $V=k((z))^{\oplus r}$ с фильтрацией $V(n)=z^{n} k[[z]]^{\oplus r}$. Пусть $V_{1}=V(0)$.

Теорема 1. Существует такое каноническое отображение $\Phi_{1}: \mathscr{M}_{1} \rightarrow\{$ векторные подпространства $A \subset k((z)), W \subset V\}$, что

(i) когомологии комплексов $A \oplus k[[z]] \rightarrow k((z)), W \oplus V_{1} \rightarrow V$ изоморфньь соответственно $H^{\cdot}\left(C, \mathscr{O}_{C}\right)$ и $H^{\cdot}(C, \mathscr{F})$,

(ii) если $(A, W) \in \operatorname{Im} \Phi_{1}$, mo $A \cdot A \subset A, A \cdot W \subset W$,

(iii) если $m, m^{\prime} \in \mathscr{M}_{1}$ и $\Phi_{1}(m)=\Phi_{1}\left(m^{\prime}\right)$, то $m$ изоморфен $m^{\prime}$.

Если $m=\left(C, P, z, \mathscr{F}, e_{P}\right) \in \mathscr{M}_{1}$, то можно положить $A:=\Gamma\left(C-P, \mathscr{O}_{C}\right)$, $W:=\Gamma(C-P, \mathscr{F})$.

Покажем, как эта конструкция связана с иерархией КП. Пусть $r=1$. Обозначим через $\operatorname{Gr}(V)$ множество подпространств $W$ в $V$, удовлетворяющих условию теоремы. Это (бесконечномерное) проективное многообразие, связные компоненты которого нумеруются эйлеровой характеристикой комплекса $W \oplus V_{1} \rightarrow V$. Если $W \in \operatorname{Gr}(V)$, то касательное пространство в точке $W$ имеет вид $T_{W}=$ $\operatorname{Hom}(W, V / W)$ и определено естественное отображение $\operatorname{Hom}(V, V) \rightarrow T_{W}$. Для $n \in \mathbb{Z}$ на $\operatorname{Gr}(V)$ имеется векторное поле $T_{n}$. Оно равно образу оператора умножения на $z^{-n}$ в пространстве $V$. Поля $T_{n}$ переходят в иерархию КП после применения соответствия Сато.

Пусть $E=k[[x]]\left(\left(\partial^{-1}\right)\right)$ - кольцо формальных псевдодифференциальных операторов с регулярными коэффициентами. Имеется разложение $E=E_{+} \oplus E_{-}$ на дифференциальные и вольтерровы операторы. Тогда иерархия КП в лаксовой форме имеет вид $\partial_{n} L=\left[\left(L^{n}\right)_{+}, L\right]$, где $L \in \partial+E_{-}=: E^{\prime}$. Множество $G=1+E_{-}$ будет группой, также несущей векторные поля $\partial_{n} S=-\left(S \partial^{n} S^{-1}\right)_{-} S$. Отображение $E \rightarrow E / E x=V$ (если отождествить образ оператора $\partial^{-1}$ с $z$ ) дает линейное действие кольца $E$ на $V$ и затем на $\operatorname{Gr}(V)$. Имеем диаграмму биекций (соответствие Сато)

$$
E^{\prime} \stackrel{\varphi}{\longleftarrow} G / G_{0} \stackrel{\psi}{\longrightarrow} \operatorname{Gr}_{+}(V) / k[[z]]^{*},
$$

*Работа поддержана грантом РФФИ, проект 00-01-00170. 
где $G_{0}=G \cap k\left(\left(\partial^{-1}\right)\right), \varphi(S)=S \partial S^{-1}, \psi(S)=S^{-1}\left(W_{0}\right)\left(W_{0}=k\left[z^{-1}\right] \in \operatorname{Gr}(V)\right)$ и $\mathrm{Gr}_{+}(V)$ - открытое подмножество (большая клетка). Отображение $\varphi$ переводит поля $\partial_{n} S$ в иерархию КП, а отображение $\psi$ переносит $\partial_{n} S$ в $T_{n}$.

Соответствие Кричевера $\Phi_{1}$ позволяет построить интегральные многообразия в $X=\operatorname{Gr}(V) / k[[z]]^{*}$. Зафиксируем $C, P, z$. Тогда образ $\Phi_{1}\left(C, P, z, \mathscr{F}, e_{P}\right)$ в $X$ не зависит от $e_{P}$ и при переменном обратимом пучке $\mathscr{F}$ заметает обобщенный якобиан $\operatorname{Jac}(C)$ кривой $C$. Это вытекает из точной последовательности $A+V_{1} \rightarrow$ $k((z)) \rightarrow T_{X, W}$, в которой $k((z)) / A+V_{1}=H^{1}\left(C, \mathscr{O}_{C}\right)$ - касательное пространство к обобщенному якобиану кривой $C$ и все векторные поля $T_{n}$ принадлежат образу $H^{1}\left(C, \mathscr{O}_{C}\right)$ в $T_{X, W}$ (см. [7]). Если $C-$ проективная прямая с одной двойной точкой, то $\operatorname{Jac}(C)=k^{*}$, и мы получаем 1 -солитонное решение.

Можно предположить, что аналогичные конструкции имеют место и для более высоких размерностей (относительно колец ПДО см. [11]). Здесь мы предлагаем обобщение отображения Кричевера $\Phi_{1}$ на случай алгебраических поверхностей.

Пусть $X-$ неприводимая проективная поверхность над полем $k, \eta$ - ее общая точка, $K$ - поле рациональных функций на $X, \widehat{\mathscr{O}}_{x}-$ пополненное локальное кольцо в точке $x \in X, K_{x, D}$ - двумерное локальное поле, отвечающее флагу $x \in D \subset X[9,5,4], \widehat{\mathscr{O}}_{x, D}-$ его кольцо дискретного нормирования, $K_{x}=K \widehat{\mathscr{O}}_{x}$ и $K_{D}$ - поле отношений локального кольца дивизора $D$ на $X$. Зафиксируем на $X$ неприводимую проективную кривую $C$ и точку $P \in C$, гладкую на $C$ и $X$. Пусть $\mathscr{F}-$ пучок без кручения на $X$ и $\widehat{\mathscr{F}}_{x}, \widehat{\mathscr{F}}_{C}-$ его пополненные слои соответственно в точке $x$ и вдоль кривой $C$. Если $x \in C$, то положим $B_{x}(\mathscr{F})=$ $\bigcap_{D \neq C}\left(\left(\widehat{\mathscr{F}}_{x} \otimes K_{x}\right) \cap\left(\widehat{\mathscr{F}}_{x} \otimes \widehat{\mathscr{O}}_{x, D}\right)\right)$, где пересечение берется внутри $\widehat{\mathscr{F}}_{x} \otimes K_{x}$, $B_{C}(\mathscr{F})=\left(\widehat{\mathscr{F}}_{C} \otimes K_{C}\right) \cap\left(\bigcap_{x \neq P} B_{x}(\mathscr{F})\right)$, где пересечение берется внутри $\widehat{\mathscr{F}}_{x} \otimes K_{x, C}$, $A_{C}(\mathscr{F})=B_{C}(\mathscr{F}) \cap \widehat{\mathscr{F}}_{C}$ и $A(\mathscr{F})=\widehat{\mathscr{F}}_{\eta} \cap\left(\bigcap_{x \in X-C} \widehat{\mathscr{F}}_{x}\right)$.

Теорема 2. Предположим, ито дивизор $C$ обилен на $X$. Тогда адельный комплекс $\widehat{\mathscr{F}}_{\eta} \oplus \prod_{D} \widehat{\mathscr{F}}_{D} \oplus \prod_{x} \widehat{\mathscr{F}}_{x} \rightarrow \prod_{D}^{\prime}\left(\widehat{\mathscr{F}}_{D} \otimes K_{D}\right) \oplus \prod_{x}^{\prime}\left(\widehat{\mathscr{F}}_{x} \otimes K_{x}\right) \oplus \prod_{x \in D}^{\prime}\left(\widehat{\mathscr{F}}_{x} \otimes\right.$ $\left.\widehat{\mathscr{O}}_{x, D}\right) \rightarrow \prod_{x \in D}^{\prime} \widehat{\mathscr{F}}_{x} \otimes K_{x, D}$ для пучка $\mathscr{F}$ квазиизоморфен комплексу $A(\mathscr{F}) \oplus$ $A_{C}(\mathscr{F}) \oplus \widehat{\mathscr{F}}_{P} \rightarrow B_{C}(\mathscr{F}) \oplus B_{P}(\mathscr{F}) \oplus\left(\widehat{\mathscr{F}}_{P} \otimes \widehat{\mathscr{O}}_{P, C}\right) \rightarrow \widehat{\mathscr{F}}_{P} \otimes K_{P, C}$.

Пример. Пусть $X=\mathbf{P}_{2} \supset C=\mathbf{P}_{1} \ni P$. Выберем такие однородные координаты $\left(x_{0}: x_{1}: x_{2}\right)$, что $C=\left(x_{0}=0\right), P=\left(x_{0}=x_{1}=0\right)$ и $U=X-C=$ Spec $k[x, y]$ с $x=x_{1} / x_{0}, y=x_{2} / x_{0}$. Тогда $k(C)=k(y / x)$ и $x^{-1}-$ последний параметр каждого двумерного локального поля $K_{Q, C}$ с $Q \neq P$. Имеем тогда $K_{P, C}=k((u))((t))$ (поле итерированных рядов Лорана), $u=x y^{-1}, t=y^{-1}$, $\widehat{\mathscr{O}}_{P, C}=k((u))[[t]]$ и $\widehat{\mathscr{O}}_{P}=k[[u, t]]$ и для пучка $\mathscr{F}=\mathscr{O}_{X}$ легко находим, что $B_{P}=k[[u]]((t)), B_{C}=k\left[u^{-1}\right]\left(\left(u^{-1} t\right)\right), A=\Gamma\left(U, \mathscr{O}_{X}\right)=k\left[u t^{-1}, t^{-1}\right]$ и $A_{C}=$ $k\left[u^{-1}\right]\left[\left[u^{-1} t\right]\right]$.

Для случая алгебраической поверхности соответствующие данные имеют вид $\mathscr{M}_{2}=\left\{\left(X, C, P,\left(z_{1}, z_{2}\right), \mathscr{F}, e_{P}\right)\right\}$, где $X-$ неприводимая проективная поверхность над полем $k, C \subset X-$ проективная неприводимая кривая над $k, P \in C-$ точка, гладкая на $X$ и $C, z_{1}, z_{2}$ - такие формальные локальные параметры в $P$, что $\left(z_{2}=0\right)=C$ около $P$, $\mathscr{F}-$ пучок без кручения ранга $r$ на $X$ и $e_{P}-$ тривиализация пучка $\mathscr{F}$ в $P$. Тогда мы имеем $\widehat{\mathscr{O}}_{X, P}=k\left[\left[z_{1}, z_{2}\right]\right], K_{P, C}=k\left(\left(z_{1}\right)\right)\left(\left(z_{2}\right)\right)$ и $\widehat{\mathscr{F}}_{P}=\widehat{\mathscr{O}}_{P} e_{P}=\widehat{\mathscr{O}}_{P}^{\oplus r}$. В поле $K=k\left(\left(z_{1}\right)\right)\left(\left(z_{2}\right)\right)$ имеются следующие фильтрации 
и подпространства: $K_{02}=k\left[\left[z_{1}\right]\right]\left(\left(z_{2}\right)\right), K_{12}=k\left(\left(z_{1}\right)\right)\left[\left[z_{2}\right]\right], K(n)=z_{2}^{n} K_{12}$. Они переносятся на пространство $V=K^{\oplus r}$.

Tеорема 3. Пусть $C$ - гиперплоское сечение. Существует такое каноническое отображение $\Phi_{2}: \mathscr{M}_{2} \rightarrow\{$ векторные подпространства $B \subset K, W \subset V\}$, umo

(i) $\partial л я$ всех $n$

$$
\begin{aligned}
& \frac{B \cap K(n)}{B \cap K(n+1)} \oplus \frac{K_{02} \cap K(n)}{K_{02} \cap K(n+1)} \longrightarrow \frac{K(n)}{K(n+1)}, \\
& \frac{W \cap V(n)}{W \cap V(n+1)} \oplus \frac{V_{02} \cap V(n)}{V_{02} \cap V(n+1)} \longrightarrow \frac{V(n)}{V(n+1)}
\end{aligned}
$$

- фредгольмовы комплексы индексов соответственно $\chi\left(C, \mathscr{O}_{C}\right)+n C . C$ u $\chi\left(C,\left.\mathscr{F}\right|_{C}\right)+n C . C$

(ii) когомологии комплексов $\left.\left(B \cap K_{02}\right) \oplus\left(B \cap K_{12}\right) \oplus\left(K_{02}\right) \cap K_{12}\right) \rightarrow B \oplus K_{02} \oplus$ $\left.K_{12} \rightarrow K u\left(W \cap V_{02}\right) \oplus\left(W \cap V_{12}\right) \oplus\left(V_{02}\right) \cap V_{12}\right) \rightarrow W \oplus V_{02} \oplus V_{12} \rightarrow V$ изоморфны соответственно $H^{\cdot}\left(X, \mathscr{O}_{X}\right)$ и $H^{\cdot}(X, \mathscr{F})$;

(iii) если $(B, W) \in \operatorname{Im} \Phi_{2}$, mo $B \cdot B \subset B, B \cdot W \subset W$;

(iv) для всех $n$ отображение

$$
\begin{aligned}
\left(C, P,\left.z_{1}\right|_{C},\left.\mathscr{F}(-n C)\right|_{C},\left.e_{P}(-n)\right|_{C}\right) \longmapsto & \left(\frac{B \cap K(n)}{B \cap K(n+1)} \subset \frac{K(n)}{K(n+1)}=k\left(\left(z_{1}\right)\right),\right. \\
& \left.\frac{W \cap V(n)}{W \cap V(n+1)} \subset \frac{V(n)}{V(n+1)}=k\left(\left(z_{1}\right)\right)^{\oplus r}\right)
\end{aligned}
$$

coвnadaem с $\Phi_{1}$

(v) если пучок $\mathscr{F}$ локально свободен и $X$ - поверхность Коэна-Маколея и если $\Phi_{2}(m)=\Phi_{2}\left(m^{\prime}\right), m, m^{\prime} \in \mathscr{M}_{2}$, то $m$ изоморфен $m^{\prime}$.

Если $m=\left(X, C, P,\left(z_{1}, z_{2}\right), \mathscr{F}, e_{P}\right) \in \mathscr{M}_{2}$, то для определения отображения $\Phi_{2}$ положим $B=B_{C}\left(\mathscr{O}_{X}\right), W=B_{C}(\mathscr{F}), \Phi_{2}(m)=(B, W)$. Доказательство см. в [12]. Заметим, что свойство (v) означает, что поверхность $X$ и пучок $\mathscr{F}$ можно восстановить по данным, связанным только с бесконечно малой окрестностью кривой $C$. Это свойство не переносится на произвольные пучки без кручения на $X$. Дальнейшее обобщение соответствия Кричевера на многообразия любой размерности было недавно предложено Д. В. Осиповым [8].

\section{ЛИТЕРАТУРА}

1. Álvarez Vásquez A., Munoz Porras J. M., Plaza Martín F. J. alg-geom/9606009. 2. Arbarello E., De Concini C., Kac V. G., Procesi C. Comm. Math. Phys., 117, 1-36 (1988). 3. Ben-Zvi D., Frenkel E. alg-geom/9802068. 4. Fimmel T., Parshin A. N. Introduction to the Higher Adelic Theory, Preprint, Moscow, 1996. 5. Huber A. Abh. Math. Sem. Univ. Hamburg, 61, 249-273 (1991). 6. Кричевер И. М. УМН, 32, 185-214 (1977). 7. Mulase M. Int. J. Math., 1, 293-342 (1990). 8. Osipov D. V. math/0003188. 9. Паршин A. H. Изв. АН СССР, сер. матем., 40, 736-773 (1976). 10. Паршин А. Н. В кн.: Гильберт Д. Избранные труды, т. 2. Факториал, М., 1998, с. 535-538. 11. Паршин А. Н. Труды МИРАН, 224, 266-280 (1999). 12. Parshin A. N. alg-geom/9911097. 13. Quandt I. alg-geom/9606015. 14. Segal G., Wilson G. Publ. Math. IHES, 80, 301-342 (1985). 